\title{
STABILITY ANALYSIS OF THE LAMINAR BOUNDARY LAYER FLOW
}

\author{
${ }^{1}$ Nazma Parveen and ${ }^{2}$ Md. M. K. Chowdhury \\ ${ }^{12}$ Department of Mathematics, Bangladesh University of Engineering and Technology, \\ Dhaka 1000, Bangladesh \\ E-mail: nazma@math.buet.ac.bd
}

Received 05.09.06

Accepted 19.04.08

\begin{abstract}
In this paper, stability analysis of incompressible laminar boundary layer flow is presented. For this approach, the partial differential equation is converted to ordinary differential equation by suitable approximation. The implicit finite difference scheme is used to find the point of separations of the boundary layer equations. The finite difference equations for the given flow at each longitudinal position form a linear set with a tridiagonal coefficient matrix. To ensure the correct results, the methods are checked with standard flows like flow past circular cylinder, Howarth's linear decelerating flows. These methods are demonstrated to compute accurately the separation points of several flows for which comparisons are made with previously published results. Then various series are tested with computer codes. At last, the stability diagram for plane poiseuille flow is shown.
\end{abstract}

Key words: Stability, finite difference scheme, point of separation

\section{Introduction}

The stability investigation is based on the assumption that laminar flows are affected by certain small disturbance. Then the decisive question in this connection arises as to whether the disturbances increase or die out with time. If the disturbances decay with time, the main flow is considered stable, on the other hand, if the disturbances increase with time the flow is considered unstable, and there exists the possibility of transition to a turbulent pattern. In this way a theory of stability is created. The general concept of stability has been discussed elaborately by Cunningham (1963). The discussion always boils down to one question: Can a given physical state with stand a disturbance and still return to its original state? If so, it is stable. If not, that particular state is unstable. It is the job of the stability analyst to test the effect of a particular disturbance. Boundary later theory is one of those inventions that allow a giant step in understanding to be taken.

To determine the separation point, the usual procedure is to apply numerical methods to the governing partial differential equations, compute the full-field solution, and thereby obtain the stream wise station at which the wall shear stress becomes zero. There has been a rapid development of numerical techniques in recent years especially in finite difference schemes.

For laminar boundary layer flow, the velocity profiles are geometrically similar and reduce to a single curve if $u / U_{0}$ is plotted against a dimensionless y co-ordinate 
$\eta=y \sqrt{u / v_{0} x}$. This is well known Blasius profile. The geometrical similarity is maintained, regardless of the Reynolds number of the flow or of the local skin friction. Primitive variable approach has been used to model the Navier-Stokes equations. By this method one can see what is going on in boundary layer? For reason of efficient computation, the most popular codes use transformed variables- stream function coordinates, Falkner- Skan stretching.

The problem of laminar boundary layer with pressure gradient has been studied by Doss [3]. A similar implicit technique was developed independently for the boundary equations in physical coordinates by Flugge-Lotz and Blottner [6]. The boundary layer equations transformed with the Howarth Dorodnitsyn relation was also investigated by Flugge-Lotz and Blottner. The partial differential equations reduce to ordinary differential equations at the tip of the body or at stagnation point. The solution of these ordinary differential equations provides initial conditions for a finite difference solution, which can start at the beginning of the body. In the implicit finite difference procedure developed by Blottner [7], it was assumed that both the normal and tangential velocity components are known from similarity solutions across the boundary layer.

In this paper primitive variable approach is considered to solve the boundary layer equations. As of Blottner, at the starting point of solution, the boundary layer equations are transformed to similarity variables. The partial differential equation is converted into ordinary differential equation by suitable approximation. The boundary layer equations are then solved by an implicit finite difference scheme. The scheme is demonstrated to compute accurately the separation points of several flows for which comparison with previously published results are possible. The stability of laminar boundary layer flow is also shown in this work.

\section{Governing Equations}

Two-dimensional incompressible flow by assuming the wall to be flat and coinciding with the $x$-direction, the $y$-axis being perpendicular to it is considered. Now a day, the most widely used CFD models of viscous flow use the direct or primitive variables $(u, v)$. Let's consider for simplicity, the two dimensional (2-D), incompressible equations of motion with constant transport properties as:

Continuity: $\frac{\partial u}{\partial x}+\frac{\partial v}{\partial y}=0$

$x$ momentum: $\frac{\partial u}{\partial t}+u \frac{\partial u}{\partial x}+v \frac{\partial u}{\partial y}=-\frac{1}{\rho} \frac{\partial p}{\partial x}+v\left(\frac{\partial^{2} u}{\partial x^{2}}+\frac{\partial^{2} u}{\partial y^{2}}\right)$

$y$ momentum: $\frac{\partial v}{\partial t}+u \frac{\partial v}{\partial x}+v \frac{\partial v}{\partial y}=-\frac{1}{\rho} \frac{\partial p}{\partial y}+v\left(\frac{\partial^{2} v}{\partial x^{2}}+\frac{\partial^{2} v}{\partial y^{2}}\right)$

The boundary conditions are:

$$
u=v=0 \text { for } y=0 \text {, and } u=U_{\infty} \text { for } y \rightarrow \infty
$$


The later two equations are parabolic in time. Thus the variables $(u, v)$ are computed in the same manner in CFD model.

The 2-D, steady, incompressible boundary layer equations, according to Prandtl, are given by:

$$
\begin{aligned}
& \frac{\partial u}{\partial x}+\frac{\partial v}{\partial y}=0 \\
& u \frac{\partial u}{\partial x}+v \frac{\partial u}{\partial y}=-\frac{1}{\rho} \frac{d p}{d x}+v\left(\frac{\partial^{2} u}{\partial y^{2}}\right)
\end{aligned}
$$

with the boundary conditions:

$$
\begin{array}{ll}
y=0 & u=0, v=0 \\
y=\infty & u=U(x)
\end{array}
$$

Here $d p / d x$ is used rather than $\partial p / \partial x$ to denote the pressure gradient. This is because $\partial p / \partial y=0$ across the boundary layer. The pressure ceased to be an unknown function and can now be evaluated from the potential flow solution for the body with the aid of the Bernoulli equation. Then equation (6) becomes

$$
u \frac{\partial u}{\partial x}+v \frac{\partial u}{\partial y}=U \frac{d U}{d x}+v\left(\frac{\partial^{2} u}{\partial y^{2}}\right)
$$

\section{Method of Solution}

\subsection{Implicit Finite Difference Method}

By using finite difference method the equations (8) and (5) respectively becomes

$$
\begin{aligned}
& -\alpha u_{m+1, n+1}-\alpha u_{m+1, n-1}+(1+2 \alpha) u_{m+1, n}=u_{m, n}+\frac{U_{m+1}^{2}-U_{m}^{2}}{2 u_{m, n}}-\beta\left[u_{m, n+1}-u_{m, n-1}\right] \\
& v_{m+1, n}=v_{m+1, n-1}-\frac{\Delta y}{2 \Delta x}\left[u_{m+1, n}-u_{m, n}+u_{m+1, n-1}-u_{m, n-1}\right]
\end{aligned}
$$

where $m$ and $n$ are number of locations in $x$ and $y$ direction respectively.

where, $\alpha=\frac{\Delta x}{\Delta y^{2} u_{m, n}}$ and $\beta=\frac{v_{m, n} \Delta x}{2 u_{m, n} \Delta y}$.

The equations (9) and (10) have to be simultaneously solved for $u_{m+1, n}$ and $v_{m+1, n}$.

Equations (9) and (10) constitute satisfactory implicit laminar boundary layer.

The boundary conditions for the above method are:

Upstream condition : $\quad u_{e}$ 
No slip

$$
u_{m, 1}=v_{m, 1}=0
$$

Known initial profiles

$$
u_{1, n}, v_{1, n}
$$

\subsection{Inversion of Tri-diagonal Matrix}

Assuming that $\mathrm{n}=1$ is for the wall and $\mathrm{n}=\mathrm{n}$ is for the free stream position, equation (9) represents (N-2) numbers of equations, each with three unknown namely $u_{m+1, n-1} ; u_{m+1, n}$; and $u_{m+1, n+1}$. The set of algebraic equations can be written in tri diagonal matrix form. Such matrix can be inverted by Gauss elimination or matrix inversion process. Here in this work, Gauss elimination is used to invert the matrix.

The equation (9) can be rewritten in the form

$$
\mathrm{A}_{n} u_{m+1, n-1}+\mathrm{B}_{n} u_{m+1, n}+C_{n} u_{m+1, n+1}=D_{n}
$$

where,

$$
\begin{aligned}
& \mathrm{A}_{n}=-\alpha(m+1, n) \\
& \mathrm{B}_{n}=1+2 \alpha(m+1, n) \\
& \mathrm{C}_{n}=-\alpha(m+1, n) \\
& \mathrm{D}_{n}=\text { right hand side of the equation (9). }
\end{aligned}
$$

After solving the tri diagonal matrix, the values $u_{m+1, n}$ are to be substituted in the equation (9) to get the normal velocity at all positions across the boundary layer.

\subsection{Initial profiles}

To find the initial values of $u$ and $v$ let us assume the variables as $\psi=u_{e} \delta f(\eta)$,

where $\psi$ is the non dimensional stream function defined in the usual way as

$$
u=\frac{\partial \psi}{\partial y}=f^{\prime} u_{e} \text { and } v=-\frac{\partial \psi}{\partial x}=-f\left[u_{e} \delta\right]^{\top}+\eta u_{e} f^{\prime} \delta^{\prime} .
$$

The primes on $u_{e}$ and $\delta$ stand for differentiation with respect to $x$.

The momentum equation (8) for a steady boundary layer flow is

$$
u \frac{\partial u}{\partial x}+v \frac{\partial u}{\partial y}=u_{e} u_{e}^{\prime}+v \frac{\partial^{2} u}{\partial y^{2}} .
$$

Using (13) in (14) and after some simplification we have the following transformed equation

$$
f^{\prime \prime \prime}+1 / 2(\beta+1) f f^{\prime \prime}+\beta\left(1-f^{\prime^{2}}\right)=0 .
$$


The equation is known as Falkner-Skan equation.

Here $\eta=\frac{y}{\delta}=\frac{y}{\sqrt{\frac{v L}{u_{0}}\left(\frac{x}{L}\right)^{m+1}}}$ and $\beta= \pm \frac{x}{u_{e}} \frac{d u_{e}}{d x}$

The Falkner-Skan profiles supply most of the initial conditions like

$$
\begin{array}{ll}
\beta=1 & \text { plane stagnation point } \\
1<\beta<0 & \text { wedge half angle } \\
\beta=0 & \text { flat plate with sharp leading edge. }
\end{array}
$$

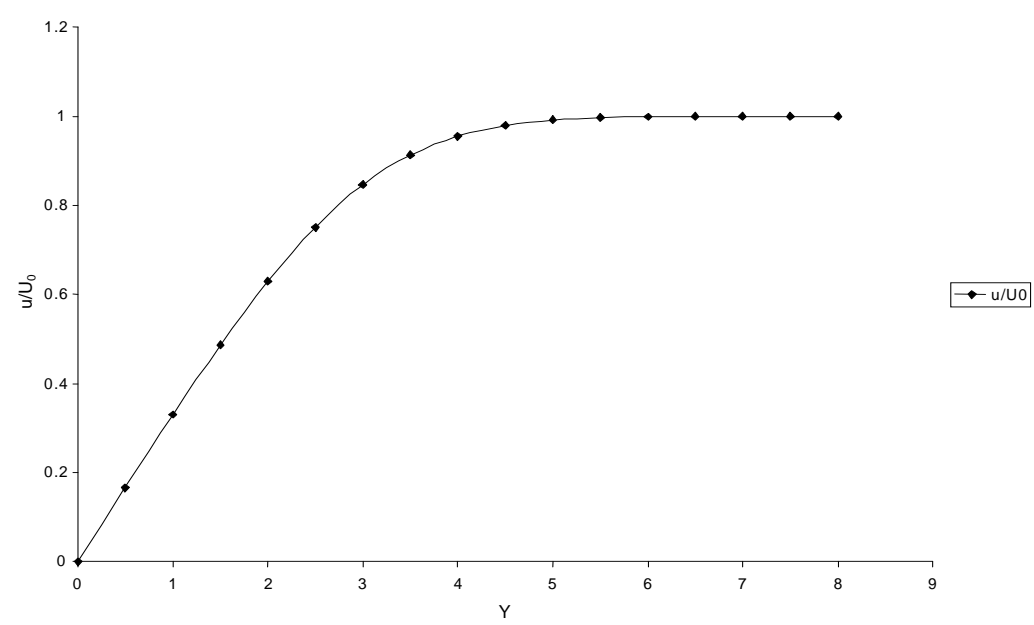

Figure 1: Velocity distribution in the boundary layer along a flat plate, after Blasius.

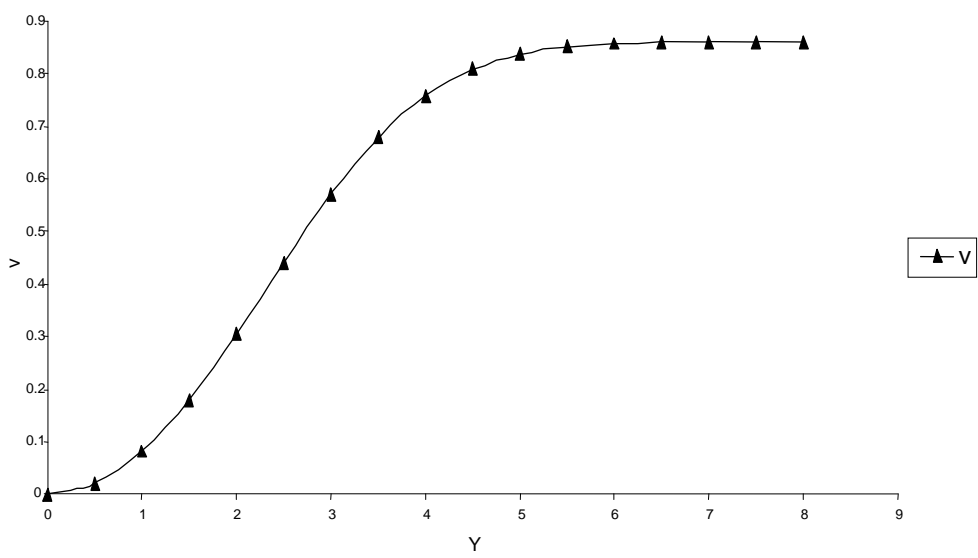

Figure 2: The transverse velocity component in the boundary layer along a flat plate. 
If the body is blunt, such as the rounded nose of an airfoil, the stagnation point solution is appreciated for starting the calculation. If the body has a pointed front then the solution corresponding to the proper wedge angle has to be used. If the body has a sharp leading edge, the solution for $\beta=0$ is used. This will be true irrespective of the pressure gradient at the leading edge. Figure 1 and figure 2 show the velocity profiles that we obtain from the Blasius equation which is a special case of Falkner-Skan equation having $\beta=0$. And the velocity profile along the flat plate figure 1 is the initial velocity profile.

\section{Test cases and Results}

\subsection{Boundary Layer Separation for Howarth Linearly Retarded Flows}

L. Blasius (1908) presented a computation method for general boundary layers with arbitrary velocity distributions of the outer flow. This was based on a series expansion of the solution in powers of $x$. It is thus called the Blasius series. The Blasius series has also been extended by L. Howarth(1935).

The family of potential flows $u(x)=\boldsymbol{U}_{0}-\mathrm{a} x^{n}(\mathrm{n}=1,2,3 \ldots \ldots .$.$) cause separation of the$ boundary layer (laminar) in a relatively short distance. In the simple case with $\mathrm{n}=1$, which was treated by L. Howarth, is another example of a boundary layer for which the velocity profiles are not similar. L. Howarth introduced in this case a new independent variable $\eta=\frac{1}{2} y \sqrt{\frac{U_{0}}{v x}}$. Another assumption is $x / L=x^{*}=\frac{a x}{U_{0}}$.

A simple decelerating non similar velocity distribution for $\mathrm{n}=1$ as given by Howarth

$$
u(x)=U_{0}(1-x / \mathrm{L})
$$

As mentioned earlier, the implicit finite difference model is used to predict the separation point for the above non-similar flow. Arbitrary values can be taken for velocity $U_{0}$ and length $\mathrm{L}$, since the results are non dimensional.

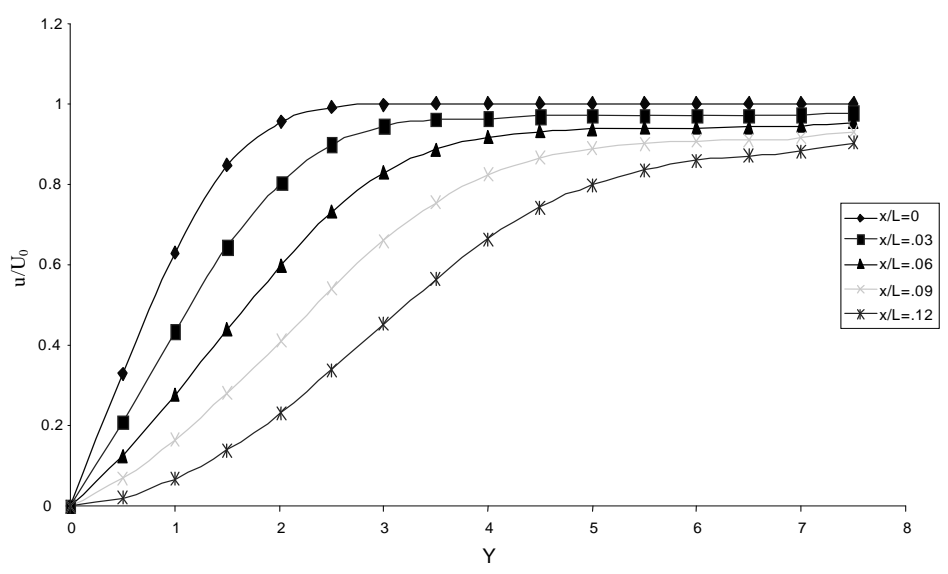

Figure 3: Velocity Profiles for Howarth flow. 


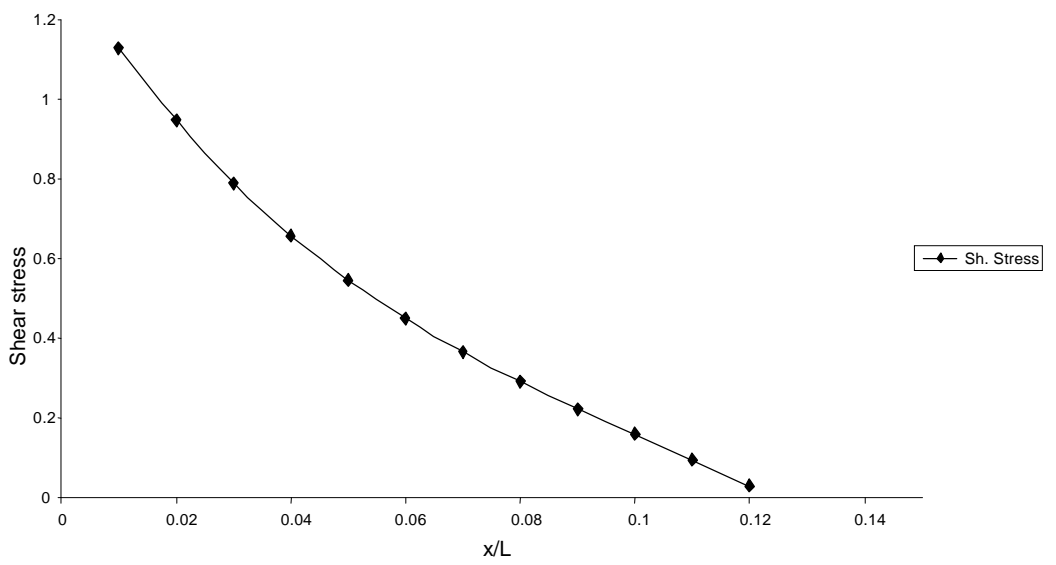

Figure 4: Shear stress distribution for Howarth flow.

The initial profiles are calculated for $\beta=0$. The step sizes for the method calculating the initial profile are $\Delta x=0.01 \& \Delta y=0.1$. For different step sizes of $\Delta x$ and $\Delta y$ there is no sensitivity difference of initial velocity profiles. Calculation of the initial profile has to be specified accurately to have the agreeable results further downstream. Figure 3 shows the velocity profiles for Howarth flow and figure 4 shows the computed values of shear stress. The profiles become increasingly $\mathrm{S}$ - shaped in the downstream and finally the separation occurs at $x / \mathrm{L} \sim 0.120$.

\subsection{Classical Analytical Solutions:}

Other classical solutions of the boundary layer equations for special $u(x)$ are:

Tani $u=U_{0}\left(1-x^{* n}\right)$

Where, $\mathrm{n}=2,4,8$ and $x^{*}=x / \mathrm{L}$

$\mathrm{n}=2$; Quadratically retarded flow

$\mathrm{n}=4 ; \quad$ Quartically retarded flow

$\mathrm{n}=8 ; \quad$ Octally retarded flow

Gortler: $\mathrm{u}=U_{0}(1-x)^{n}$

$$
\begin{aligned}
& \mathrm{n}=1 / 2,2 \\
& \mathrm{u}=U_{0}(1+x)^{n} \\
& \mathrm{n}=-1,-2
\end{aligned}
$$

Table 1 gives a comparison of the presently predicted and previously published locations of the separation points for the flows considered above. For the Howarth - Tani type of retarded flows, a graph is drawn between the separation point and the values of ' $n$ ' as shown in Figure 5. In the same manner graphs are drawn for Gortler flows for different values of ' $n$ ' as shown in Figure 6. 


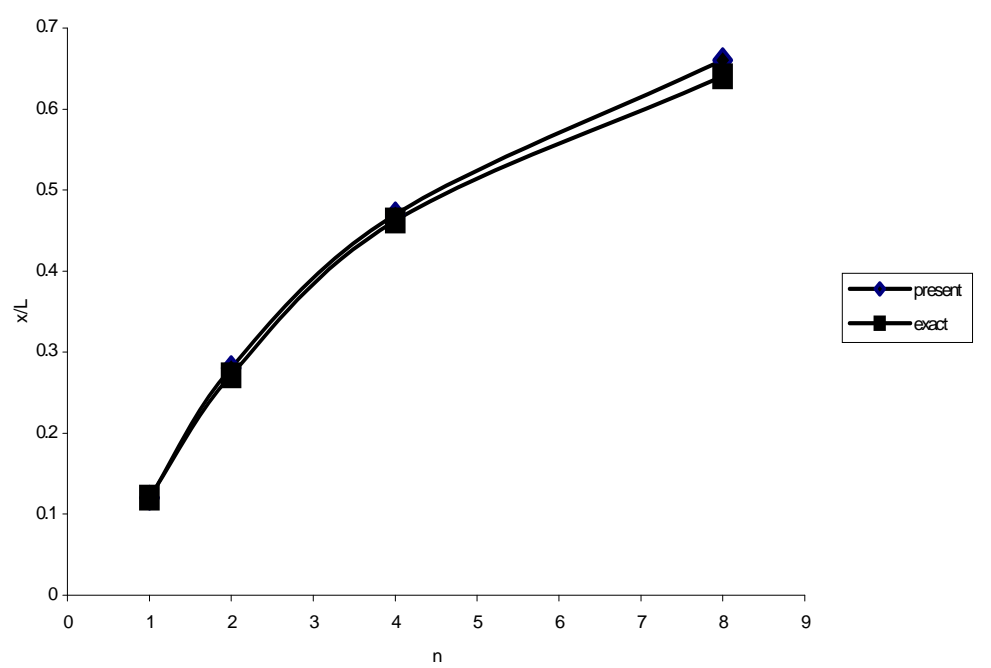

Figure 5: Comparison of separation of Howarth-Tani flows between present method and exact method for different values of exponent $n$.

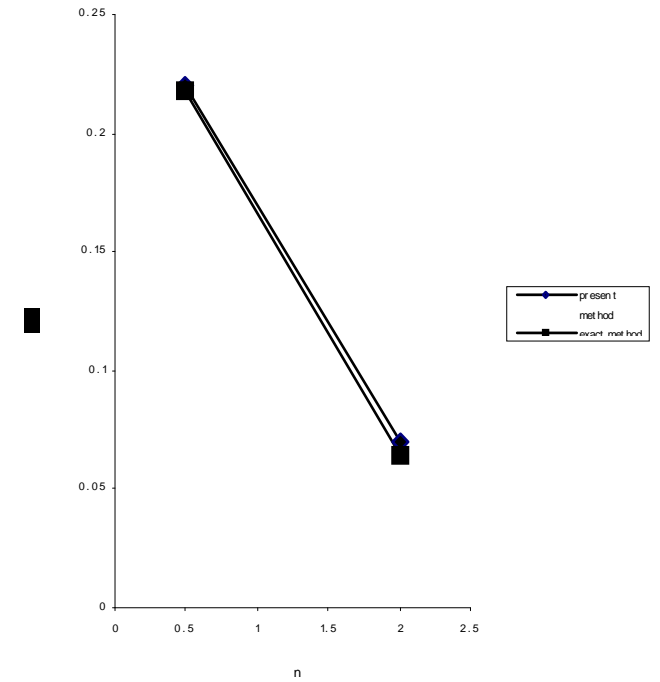

(a)

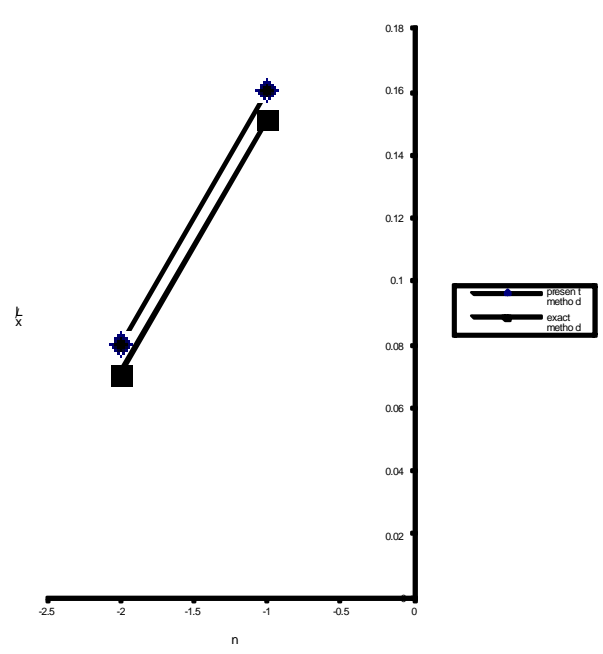

(b)

Figure 6: (a)Separation of Gortler flow [1-x $\left.{ }^{n} ; \mathrm{n}=1 / 2,2\right]$;(b): Separation of Gortler flow [ $\left.(1+\mathrm{x})^{\mathrm{n}} ; \mathrm{n}=-1,-2\right]$.

\subsection{Flow Past a Circular Cylinder}

Both the accuracy and dilemma of a bluff body boundary layer calculation are illustrated by the circular cylinder. In terms of the dimensionless are length, $x^{*}=x / \mathrm{R}$, where $\mathrm{R}$ is the cylinder radius. The ideal velocity distribution in non-viscous, irrotational flow past a circular cylinder of radius $\mathrm{R}$ and free stream velocity $U_{\infty}$ parallel to the $x$-axis is given by

$$
u(x)=2 U_{\infty} \sin (x / \mathrm{R})=2 U_{\infty} \sin \phi
$$


where $\phi$ is the angle measured from the stagnation point. Expanding $\sin (x / \mathrm{R})$ into a series and the potential flow velocity distribution is

$$
\begin{aligned}
& \frac{u}{U_{\infty}}=2 \sin x^{*}=2.0 x^{*}-\frac{2}{3 !} x^{* 3}+\frac{2}{5 !} x^{* 5} \\
& \frac{u}{U_{\infty}}=2.0 x^{*}-0.333 x^{* 3}+0.0167 x^{* 5}
\end{aligned}
$$

From the above expression, the boundary layer solutions are obtained. Separation for the above flow is predicted at an angle $x^{*}=\phi=105.82 \mathrm{deg}$. from the present method. To start the calculation for marching, the initial profiles are calculated by taking the value as, $\beta=1$, i. e. at the stagnation point. Boundary layer profiles for circular cylinder are plotted in Figure 7 in the favorable gradient for $\phi$ up to $60^{\circ}$, the profiles are strongly curved and the wall shear stresses have increased. From $60^{\circ}$ on as free stream levels off and beings to accelerate. And then the profiles become thicker and then S- shaped with an early separation. Table 1 gives the values of separation point for different types of flows. Test cases results are nearly matched with standard results.

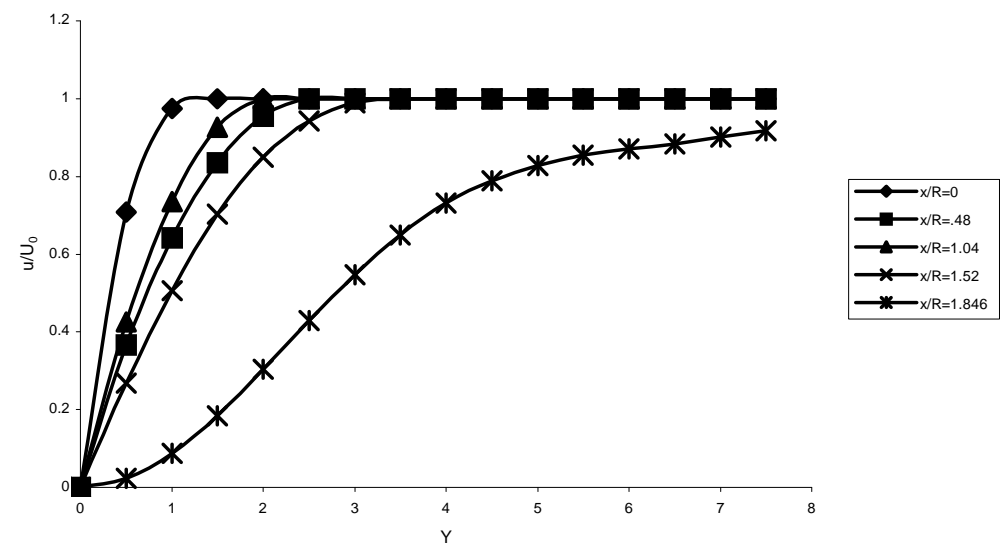

Figure 7: Velocity profiles for flow circular cylinder.

\subsection{Stability Analysis of the Laminar Boundary Layer Flow}

From Orr-Somerfeld equation we know that if $\mathrm{C}_{i}<0$, the disturbances are damped and the laminar mean flow is stable. And if $\mathrm{C}_{i}>0$, the mean flow is unstable. In Figure 8, we see the stability diagram for laminar boundary layer flow. Where $\mathrm{C}_{i}>0$, so the flow is instability sets. If we compare this stability diagram with the exact diagram, we see that the exact Reynolds number and $\alpha \mathrm{L}$ are 5767 and 1.02 respectively when $\mathrm{C}_{i}=0$. In this work the Reynolds number and $\alpha \mathrm{L}$ are 5800 and 1.02 respectively when $\mathrm{C}_{i}=0$ which are labeled in the figure 8 . 


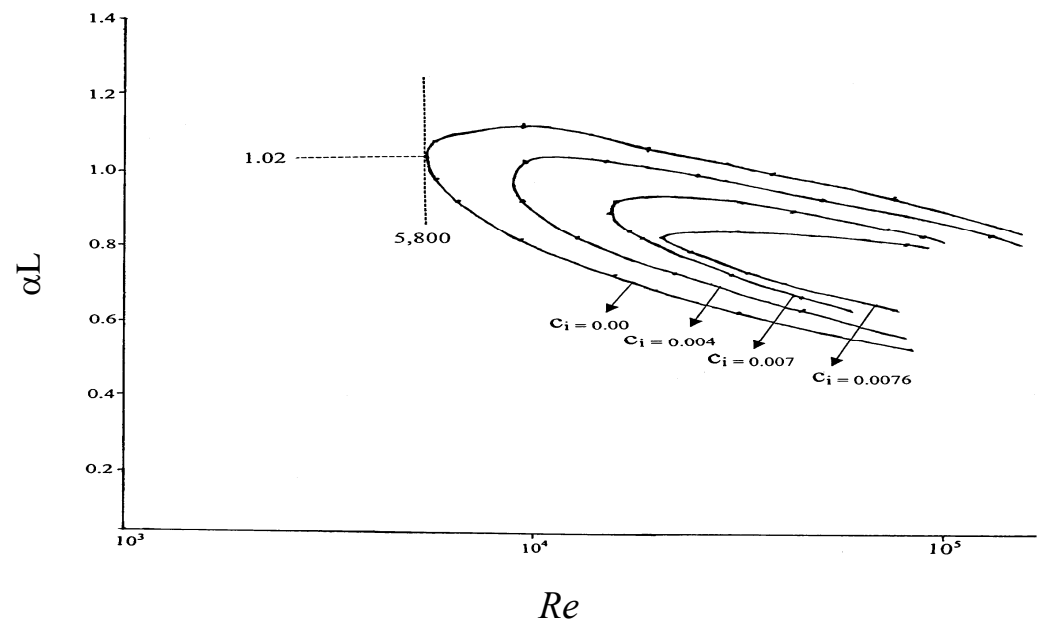

Figure 8: Stability diagram.

Table 1: Comparison of presently predicted and previously published Separation points.

\begin{tabular}{|c|c|c|c|}
\hline \multirow{2}{*}{\multicolumn{2}{|c|}{ Description of flows }} & \multicolumn{2}{|c|}{ Physical separation point } \\
\hline & & Presently predicted & Previously published \\
\hline \multicolumn{2}{|c|}{ Linearly retarded flow, $\mathrm{n}=1$} & 0.120 & 0.120 \\
\hline \multicolumn{2}{|c|}{ Quadratically retarded flow, $n=2$} & 0.280 & 0.271 \\
\hline \multicolumn{2}{|c|}{ Quartically retarded flow, $\mathrm{n}=4$} & 0.470 & 0.462 \\
\hline \multicolumn{2}{|c|}{ Octally retarded flow, $\mathrm{n}=8$} & 0.660 & 0.640 \\
\hline \multirow{4}{*}{ Gortler Flow } & $\mathrm{n}=1 / 2$ & 0.220 & 0.218 \\
\hline & $\mathrm{n}=2$ & 0.070 & 0.0637 \\
\hline & $\mathrm{n}=-1$ & 0.160 & 0.151 \\
\hline & $\mathrm{n}=-2$ & 0.080 & 0.0713 \\
\hline \multicolumn{2}{|c|}{ Potential flow past a circular cylinder } & $105.82 \mathrm{deg}$ & $104.45 \mathrm{deg}$ \\
\hline
\end{tabular}

\section{Conclusion}

The paper deals with the solution of the laminar boundary layers. We have presented the primitive variable approach as calculating tool of the boundary layer problem. The coupled equation results for circular cylinder, Howarth's linear decelerating flows etc. matching with the previously published results. It has been are shown that implicit finite difference method along with the transformations for removing singularity at the starting point is very useful in analyzing the different types of flows. In this approach visualization of the things can be done which is not possible in transformation plane like Falkner - Skan stretching, stream function coordinates.

This method is highly depending on the initial profile as well as derivation of the ordinary differential equation. The overall analysis is extremely sensitive, if the separation region is relatively small, as the method does not involve iteration process. 


\section{Nomenclature:}

$u, v \quad$ Components of the velocity

$\Delta x \quad$ Step size in ' $x$ ' direction

$\Delta y \quad$ Step size in ' $y$ ' direction

$x, y \quad$ Coordinates along and normal to the surface of the body

$f(\eta) \quad$ Function related to stream function

L Reference length

$\mu \quad$ Viscosity coefficient

$\tau \quad$ Shearing stress

$\psi \quad$ Stream function

$v \quad$ Coefficient of kinematic viscosity

$\eta \quad$ Transformed ' $y$ ' co-ordinate in the boundary layer equation

$U(x) \quad$ Non - similar velocity distribution in $x$ direction

m Exponent in free stream velocity variation of similar flow, $U=\mathrm{c} x^{m}$

$\alpha, \beta \quad$ Finite difference mesh size parameters

$u_{e} \quad$ Free stream velocity

\section{Subscripts}
$\infty$
Free stream conditions
e External conditions
$m \quad$ No of locations in $x$ direction
$n \quad$ No of locations in $y$ direction

\section{Superscripts}

Primes ( $\left.{ }^{\prime}\right)$ differentiation with respect to $\eta$

\section{REFERENCES}

1. Hermann schlicting, Boundary Layer Theory, seventh edition McGraw hill Book Company, New York, (1979).

2. Frank M. White, Viscous Fluid Flow, Second edition McGraw Hill Book Company, New York, (1991).

3. Doss, K. J. M., Calculation of Laminar Boundary Layer with Pressure Gradient, IndianInstitute of Technology, New Delhi, December, (1999).

4. Curtis F. Gerald and Patrick O. Wheatley, Applied Numerical Analysis, Fifth edition, (1994).

5. Leight, D. C. F., The Laminar Boundary Layer Equation: A Method Solution by means of an Automatic Computer, Proceedings of the Cambridge Philosophic Society, Vol.51, pp.320-322, (1955). 
6. Flugge- Lotz, I. And Blottner, F.G., Computation of Compressible Laminar Boundary Flow Including Displacement - Thickness Interaction Using Finite - Difference Methods, TR 131, Jan. 1962, Div of Engineering Mechanics, Standard Univ., for abbreviated version, see journal de Mechanique, Vol. II, No. 4 Dec. 1963.

7. Blottner, F. G., Finite Difference Methods of Solution of the Boundary Layer Equations, AIAA Journal, Vol. 8, No. 2, Feb., PP. 193-205 (1970).

8. Kendall, R. M. and Bertlett, E.P., Nonsimilar Solution of the Multicomponent Laminar Boundary layer by an Integral Matrix Method, AIAA journal, Vol. 6, No. 6, June (pp. 1089-1097 1968). 